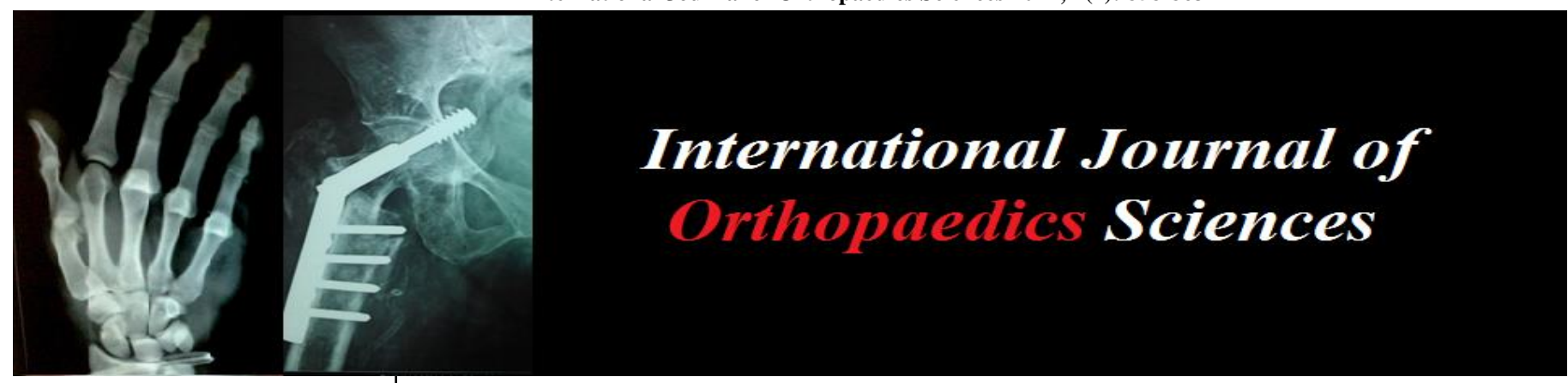

E-ISSN: 2395-1958

P-ISSN: 2706-6630

IJOS 2021; 7(1): 858-863

(C) 2021 IJOS

www.orthopaper.com

Received: 28-11-2020

Accepted: 30-12-2020

\section{Ahmet Ateş}

University of Health Sciences,

Keçiören Health Practice and

Research Center Orthopaedics

and Traumatology Department Ankara, Turkey

Yüksel Uğur Yaradılmış

Pınarbașı Mh. Sanatoryum Cd.

Ardahan Sk. No:25, Keçiören,

06380, Ankara, Turkey

Mustafa Caner Okkaoğlu

Pınarbaşı Mh. Sanatoryum Cd. Ardahan Sk. No:25, Keçören,

06380, Ankara, Turkey

\section{Erdi Özdemi}

Pınarbașı Mh. Sanatoryum Cd. Ardahan Sk. No:25, Keçiören, 06380, Ankara, Turkey

\section{İsmail Demirkale}

Pınarbaşı Mh. Sanatoryum Cd. Ardahan Sk. No:25, Keçiören, 06380, Ankara, Turkey

\section{Murat Altay}

Pınarbaşı Mh. Sanatoryum Cd. Ardahan Sk. No:25, Keçiören, 06380, Ankara, Turkey
Corresponding Author: Yüksel Uğur Yaradılmış Pınarbaşı Mh. Sanatoryum Cd. Ardahan Sk. No:25, Keciören, 06380, Ankara, Turkey

\section{Controlled femoral impaction for reduction of hip arthroplasty in high riding hips: Is it safe?}

\author{
Ahmet Ateş, Yüksel Uğur Yaradılmış, Mustafa Caner Okkaoğlu, Erdi \\ Özdemir, İsmail Demirkale and Murat Altay
}

DOI: https://doi.org/10.22271/ortho.2021.v7.i1m.2582

\section{Abstract}

Background: The subtrochanteric femoral shortening osteotomy (SFO) is a complex surgical procedure The aim of this study was to describe the novel controlled femoral impaction technique as a safe reduction method to avoid SFO during total hip arthroplasty (THA) and present its results in high dislocated hip patients who undergo THA.

Materials and Method: This retrospective study included 40 Crowe developmental dysplasia of the hip (DDH) type III/IV patients for whom SFO was not planned in preoperative templating. Patients who were expected to have $>4 \mathrm{~cm}$ lengthening according to the preoperative templating are excluded. Patients were evaluated in respect of functional results with Harris Hip Score (HHS), limb length discrepancy (LLD) and complications. Of the $40 \mathrm{DDH}$ patients who underwent THA without femoral shortening, controlled femoral cracking was performed in 20 cases while no additional procedure was required in 20 cases.

Results: There were 3 male and 37 female patients with a mean age of $53.7 \pm 9.54$ years. The mean follow-up period was 38 \pm 6.54 months (range, 24-66 months). The mean Harris Hip Score (HHS) was 45.96 (range, 38-72) preoperatively and 89.44 \pm 6.4 (range, 84-99) postoperatively. LLD was 3.4 \pm 0.7 (range, $0-4 \mathrm{~cm}$ ) $\mathrm{cm}$ preoperatively and $0.7 \pm 0.5 \mathrm{~cm}$ (range, $0-2 \mathrm{~cm}$ ) postoperatively $(\mathrm{p}<0.05)$. There was no significant difference in latest postoperative mean HSS in patients who underwent controlled femoral impaction $(88.2 \pm 6.3)$ or not $(90.3 \pm 6.5)(\mathrm{p}=0.740)$. There was no increase in complications with controlled femoral impaction.

Conclusion: Controlled femoral impaction seems to be a safe technique during THA for DDH patients where more than $4 \mathrm{~cm}$ of lengthening is not expected preoperatively. SFO could be avoided with controlled femoral impaction technique without increasing LLD or nerve palsy rate.

Keywords: high dislocated hip, without femoral shortening osteotomy, hip reduction, controlled femoral impaction, surgery technique

\section{Introduction}

Although total hip arthroplasty (THA) for high dislocated hips has good long-term survival and high patient satisfaction, it is one of the most complex orthopaedic surgeries because of the dysplastic acetabulum and femoral anatomy ${ }^{[1-3]}$. In the surgical planning of THA for high dislocated hips, there is consensus that the center of rotation of the acetabulum should be protected and therefore, the center of rotation should be formed in the true acetabulum ${ }^{[4]}$

The creation of the true center of rotation entails two major challenges including difficulty during reduction of the hip joint and the fear of nerve palsy due to lengthening of the leg. Subtrochanteric femoral osteotomy (SFO) is used successfully in THA for high dislocated hips, solving the consequences of lengthening problem by facilitating reduction of the hip and reducing the possibility of neurovascular damage ${ }^{[5,6]}$. However, SFO has its own drawbacks such as longer operating time, increased amount of bleeding, being a complex surgical procedure, and osteotomy related complications including non-union, and infection ${ }^{[7]}$.

THA without a SFO for DDH patients is a relatively minimally invasive surgical procedure compared to THA with a SFO as it shortens the operating time and decreases the blood loss. The greatest advantage of the THA without SFO is that it can eliminate leg length discrepancy (LLD). In recent years, there has been an increase in studies with a large number of patients treated with the THA without SFO procedure in high dislocated hips. In studies reporting the 
results of THA without SFO surgery, additional procedures such as lowered neck osteotomy, extensive soft tissue release, and iliofemoral distraction external fixation were required both for reduction of the hip and protecting the hip biomechanics ${ }^{[8-12]}$. To increase the feasibility of THA without SFO in patients with a high dislocated hip, and to protect the hip biomechanics of patients with abnormal bony anatomy, there is a clear need for the development of new procedures. The aim of this study was to describe our novel controlled femoral impaction technique as a safe reduction method and to present the results of this method used in THA without a SFO in dislocated high-riding hips.

\section{Material and Method Patients and study design}

A retrospective examination was made of Crowe III/IV developmental dysplasia of the hip (DDH) patients who underwent THA between 2011 and 2018. Our study was ethically approved by the Ethical Committee of our hospital (Numara). The study was conducted in accordance with the principles of the Declaration of Helsinki. The study included 40 Crowe III/IV patients for whom shortening was not planned in preoperative evaluation and who had at least a 2year follow-up period. Patients who were expected to have $>4 \mathrm{~cm}$ lengthening according to the preoperative templating $(n=54)$, patients with a history of surgery on the same hip $(n=3)$, severe contracture $(n=4)$, with bilateral DDH $(n=1)$ were excluded from the study. A total of 40 Crowe III/IV hips met the inclusion criteria and were included in the study.

Of the 40 patients underwent THA without SFO, controlled femoral impaction was required in 20 cases while no additional procedure was required during reduction in 20 cases. The decision for controlled femoral impaction was made when the femoral head was above the acetabular cup during reduction After adequate soft tissue release.

The amount of leg lengthening and actual LLD was determined by measuring the distance between the medial malleolus and the spina iliaca anterior superior (SIAS) of patients. The clinical outcomes of the patients were evaluated with Hip Score (HHS) both in the preoperative period and latest postoperative visit. Harris Complications were noted. . All the patients were evaluated in respect of functional results, LLD differences, operating time, and complications.

\section{Surgical technique}

\section{Planning and acetabular preparation}

Preoperative templating was performed for all the patients to determine component sizes and center of acetabulum.

The decision for SFO was made after the preoperative templating and SFO was performed during THA of the patients who were expected to have $>4 \mathrm{~cm}$ lengthening and these patients were included in the study. For the other DDH patients, a THA without SFO surgery was planned.

With the patient in the supine position on a radiolucent operating table, a direct lateral approach was made under general anesthesia. The femoral neck cut was made from the proximal border of the trochanter minor. The true acetabulum was explored by tracking the capsule then the capsule was excised. The location of the true acetabulum was confirmed with fluoroscopy, afterwards it was exposed by clearing the surrounding soft tissues and osteophytes. Placement of the acetabular component to the center of rotation of the true acetabulum and reaming was performed without medialization and avoiding medial protrusion. A cementless press-fit acetabular cup was used, and rotational stability o the cup was provided with 2 or 3 screws. The acetabular cup sizes ranged from 40 to $54 \mathrm{~mm}$, and femoral head of 22,28 or $32 \mathrm{~mm}$ sized compatible with the acetabular cup were used.

Femoral preparation and surgery technique for reduction After completion of acetabular preparation, trial femoral component was implemented. Then an attempt was made to reduce the hip joint. If the hip joint could be reduced without any difficulties, the surgery continued without any additional procedure. Controlled femoral impaction was performed when femoral head was above the acetabular cup during reduction despite adequate soft tissue release including iliopsoas tenotomy. During the controlled femoral impaction technique, a prophylactic single cable was wrapped immediately below the trochanter minor and the trial femoral stem impaction was impacted into to femur until the point allowing the reduction of the hip joint. Following the confirmation of a reliable reduction with the trial components, the real components were implanted. If a trochanter minor fissure occurred after the implantation of the real femoral component, a second cable was wrapped around the trochanter minor (6 patients required a second cable). After reduction, joint range of motion (ROM) and the tensor fascia were evaluated. Tensor fascia release was performed if there was excessive tension. A cementless femoral stem was used in all patients; according to the DORR index, a Taperloc (Biomet, Warsaw, IN, USA) or a Secur-Fit Plus Max (Stryker, Mahwah, NJ, USA) femoral stem was used. It was aimed to obtain total anteversion (acetabular+femoral) of $20^{\circ}$ $30^{\circ}$. The surgical technique of femoral impaction is presented in Figure 1.

\section{Follow-up}

Patients were mobilized on postoperative day 1 with partial weight-bearing. At the end of 1 month, full weight-bearing was permitted. Case examples were shown in Figure 2 and 3.

\section{Statistical analysis}

Data obtained in the study were analyzed statistically using SPSS version 22 software. Qualitative data were stated as numbers (n) and percentages (\%), and quantitative data as mean, minimum and maximum values. The HSS of the groups were compared with the Mann Whitney U-test. The Chi-square test was applied to determine the relationships between the complications and groups. Results were stated in a $95 \%$ confidence interval. A value of $\mathrm{p}<0.05$ was accepted as statistically significant.

\section{Results}

There were 3 males and 37 females (M/F: 1/12) with a mean age of $53.7 \pm 9.54$ years. The mean follow-up period was $38 \pm 6.54$ months (range, 24-66 months). The operated hips were left side in 23 cases and right side in 17 cases (left/right:1.5/1). The mean body mass index (BMI) of the patients was $29.5 \pm 4.21$. The demographic data of the patients are shown in Table 1.

The mean HHS was 45.96 (range, 38-72) preoperatively and $89.44 \pm 6.4$ (range, 84-99) postoperatively $(\mathrm{p}<0.05)$. LLD was $3.4 \pm 0.7 \mathrm{~cm}$ (range, $0-4 \mathrm{~cm}$ ) preoperatively and $0.7 \pm 0.5 \mathrm{~cm}$ (range, $0-2 \mathrm{~cm}$ ) postoperatively $(\mathrm{p}<0.05)$. None of the patients had LLD of $>2 \mathrm{~cm}$ in the postoperative period (Table 1 ).

There was no significant difference in latest postoperative mean HSS in patients who underwent controlled femoral impaction $(88.2 \pm 6.3)$ or not $(90.3 \pm 6.5)(\mathrm{p}=0.740)$.

LLD at the final follow-up examination was determined as 
$0.71 \pm 0.4 \mathrm{~cm}$ in patients with controlled femoral impaction and $0.69 \pm 0.4$ (range, 86-99) in patients without controlled femoral impaction $(\mathrm{p}=0.940)$.

No statistically significant difference was determined between the groups in respect of operating time, the need for blood transfusion, dislocation, infection and neurological damage ( $\mathrm{p}=0.624, \mathrm{p}=0.746, \mathrm{p}=1.00, \mathrm{p}=1.00, \mathrm{p}=0.556$, respectively).

(Table 2)

Permanent sciatic nerve palsy developed in 1 patient where controlled femoral impaction was not performed. Sciatic nerve exploration performed for this patient and it was revealed a widespread fibrosis in the nerve sheath. Tendon transfer was performed to this patient at the end of 18 months because of the drop foot. In the patients with dislocation $(n=2)$, revision THA was performed with contrained acetabular cup.

\section{Discussion}

For many years, successful results have been obtained with the SFO procedure in high dislocated hips ${ }^{[13,14]}$. However, in patients who underwent SFO, such problems could be encountered: non-union at the osteotomy, infection, dislocation and reduced survival of thefemoral stem in addition to a longer operating time and increased blood loss $[15,16]$. Therefore, there is increasing interest in orthopaedic surgeons for performing THA without SFO procedures. In the current study, high functional scores were obtained with controlled femoral impaction technique as well as comparable complication rates including sciatic nerve damage, dislocation and infection.

Current literature has shown that the functional scores of THA without SFO procedures can be just as good as those of surgical procedures with SFO, and some studies have obtained even better results ${ }^{[7,17,18]}$. Mei XL et al. compared the results of patients underwent THA with or without SFO and reported higher HHS in the patient without SFO. In a study by Li et al. demonstrated that THA without SFO yields shorter operative time and reduced need for blood transfusion compared to THA with SFO in Crowe type IV patients ${ }^{[7]}$. Considering the existing literature and the current study, THA without SFO procedure in high dislocated hip is a successful surgical technique, which not only shortens operating time and facilitates the surgery, but also reduces morbidity.

One of the most important advantages of the THA without SFO procedure is that it allows better adjustment of LLD. Although limping and LLD is a significant postoperative complaint in high dislocated hip patients with the use of the THA without SFO procedure, LLD could be corrected significantly [8-11]. In a study by Chen et al., THA without SFO procedure was shown to reduce gluteal limping together with correction of LLD ${ }^{[19]}$. In a series of 38 THA procedures without SFO, Kawai et al. reported a mean LLD of $3.2 \mathrm{~cm}$ (range, $1-5.1 \mathrm{~cm}$ ) preoperatively and $0.6 \mathrm{~cm}$ (range, $0-1.8 \mathrm{~cm}$ ) postoperatively ${ }^{[8]}$. Similar to the Kawai study, $\mathrm{Wu}$ et al. reported LLD correction from $4.3 \mathrm{~cm}$ (range, $2.5-5.5 \mathrm{~cm}$ ) preoperatively to $1.3 \mathrm{~cm}$ (range, $0-1.6 \mathrm{~cm}$ ) postoperatively ${ }^{[10]}$. However, the amount of acceptable LLD remains unclear. Benedetti et al. showed that LLD between 1-20 mm does not impair symmetry, thus, hip kinematics are provided ${ }^{[20]}$. In the current study, there were no patients having LLD over $2 \mathrm{~cm}$ in both groups, and a significant improvement was seen in the
LLD both groups as well.

In operations when SFO is not performed, the patient must be thoroughly evaluated in respect of the possibility of nerve damage and excessive soft tissue tension. The most feared complication of THA without SFO is sciatic nerve palsy which could be develop due to the lengthening of the leg. If the leg lengthening is expected to be $>4 \mathrm{~cm}$, the SFO is recommended to prevent nerve palsy [21, 22]. Kong et al. recommended the use of neuromonitorisation when necessary to protect against neurological complications ${ }^{[23]}$. The authors also recommended that femoral shortening should be performed when reduction of the true acetabulum is not possible. In the current study, THA without SFO procedure was applied to patients where $>4 \mathrm{~cm}$ lengthening was not expected, but osteotomy decision could also be made after consideration of additional intraoperative reduction methods.

Hip reduction without SFO requires surgical experience and the surgeons mostly develop their own methods. The first stage in reduction is the use of muscle relaxants, for which Yan et al. recommended the intraoperative administration of additional rocuronium ${ }^{[12]}$. In the current study, all the procedures were performed under general anesthesia and with muscle relaxants. Another advantage of general anesthesia over regional anesthesia is that it allows the evaluation of nerve examination in the early period during patient recovery. As a more invasive reduction method, Kawai et al. applied an additional femoral neck cut extending to the level of the trochanter minor in 38 patients and obtained successful results ${ }^{[8]}$. Wu et al. performed additional soft tissue release to patients with severe contracture and were able to obtain reduction with no shortening and up to $7 \mathrm{~cm}$ lengthening ${ }^{[10]}$. Lai et al. performed THA without shortening by providing lengthening with the aid of an iliofemoral external fixator. As this method was more invasive and required two surgeons, it was reported to be less favorable than other methods ${ }^{[11]}$. In a recent study, Li et al. described a more minimally invasive reduction method with a Hohmann retractor [24]. The advantage of the novel controlled femoral impaction technique is it can be applied in a controlled manner according to the degree of intraoperative reduction.

The main limitation of this study that the number of patients was low, and the study was retrospective. Further studies are recommended for evaluation with gait analysis of varying lower extremity biomechanics after THA without shortening. A strong aspect of the current study was that all the operations were performed by a single surgeon using the same procedure.

In conclusions, patients where more than $4 \mathrm{~cm}$ of lengthening is not expected preoperatively, THA could be successfully managed without a SFO. The controlled femoral impaction technique seems to be safe method as it does not increase LLD or nerve palsy rate, shortens operating time and provides less blood loss.

\section{Declaration of conflicting interests}

The authors declared no conflicts of interest with respect to the authorship and/or publication of this article.

\section{Funding}

The authors received no financial support for the research and/or authorship of this article. 
Table 1: Demographic data of the patients without shortening femoral osteotomy

\begin{tabular}{|c|c|c|c|}
\hline & Controlled femoral impaction & Without controlled femoral impaction & Total $(\mathbf{n} / \%)$ \\
\hline Hips & 20 & 20 & 40 \\
\hline Age & $51.8(32-67)$ & $54.9(21-74)$ & $53.7 \pm 9.54$ \\
\hline \multicolumn{4}{|l|}{ Gender } \\
\hline Male & 2 & 1 & $3(7.5 \%)$ \\
\hline Female & 18 & 19 & $37(92.5 \%)$ \\
\hline \multicolumn{4}{|l|}{ Side } \\
\hline Right & 9 & 8 & $17(42.5 \%)$ \\
\hline Left & 11 & 12 & $23(57.5 \%)$ \\
\hline BMI & $30.3(23-37)$ & $29(25-33)$ & $29.5 \pm 4.21$ \\
\hline \multicolumn{4}{|l|}{ Crowe } \\
\hline Type 3 & 6 & 20 & $26(65 \%)$ \\
\hline Type 4 & 14 & & $14(35 \%)$ \\
\hline \multicolumn{4}{|l|}{ Hartofiladikis } \\
\hline B & 11 & 20 & $31(77.5 \%)$ \\
\hline $\mathrm{C}$ & 9 & & $9(22.5 \%)$ \\
\hline Acetabular grafting & 2 & 4 & $6(15 \%)$ \\
\hline
\end{tabular}

Abbreviations: BMI; Body mass index

Table 2: Comparison of data obtained from patients' follow-up

\begin{tabular}{|c|c|c|c|c|}
\hline & Patients & Controlled femoral impaction & Without controlled femoral impaction & p \\
\hline Dislocation & $2(5 \%)$ & $1(5 \%)$ & $1(5 \%)$ & 1 \\
\hline Infection & 0 & 0 & 0 & 1 \\
\hline Neurological injury & $1(2.5 \%)$ & 0 & $4(5 \%)$ & 0.556 \\
\hline Harris (preoperative) & 45.96 & 47.7 & 90.3 & 0.720 \\
\hline Harris (postoperative) & 89.44 & 88.2 & 1.9 & 0.560 \\
\hline Transfusion (U/patients) & 2 & 2 & $101 \pm 10$ & 0.746 \\
\hline Mean Surgery time (minute) & $104 \pm 20$ & $109 \pm 20$ & 3.22 & 0.624 \\
\hline Mean elongation preoperative $(\mathrm{cm})$ & 3.41 & 3.63 & 0.69 & 0.234 \\
\hline Mean elongation postoperative $(\mathrm{cm})$ & 0.71 & 0.71 & 0 & 0.940 \\
\hline LLD (>2cm) & 0 & 0 & & \\
\hline
\end{tabular}

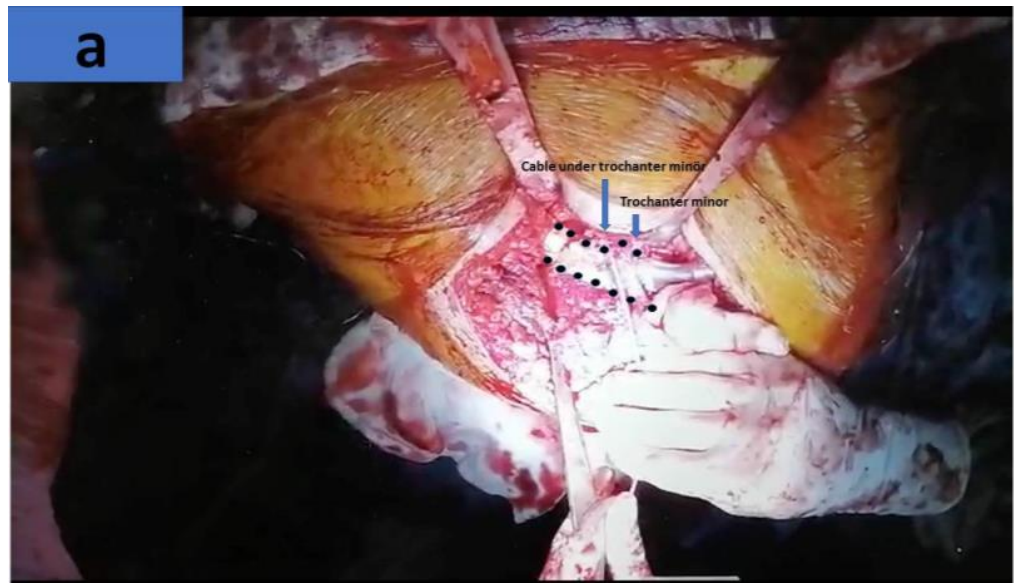

Fig 1: Controlled femoral impaction technique ( a: The prophylactic cable was wrapped around when reduction could not be obtained with the trial femoral stem after femoral preparation, b: After application of the cable, impaction of the trial femoral component was continued until the point allowing the reduction of the hip joint and reduction was confirmed with fluoroscopy, $\mathbf{c}$ : after application of the original femoral stem, a second cable was applied if a fissure occurred in the trochanter minor)
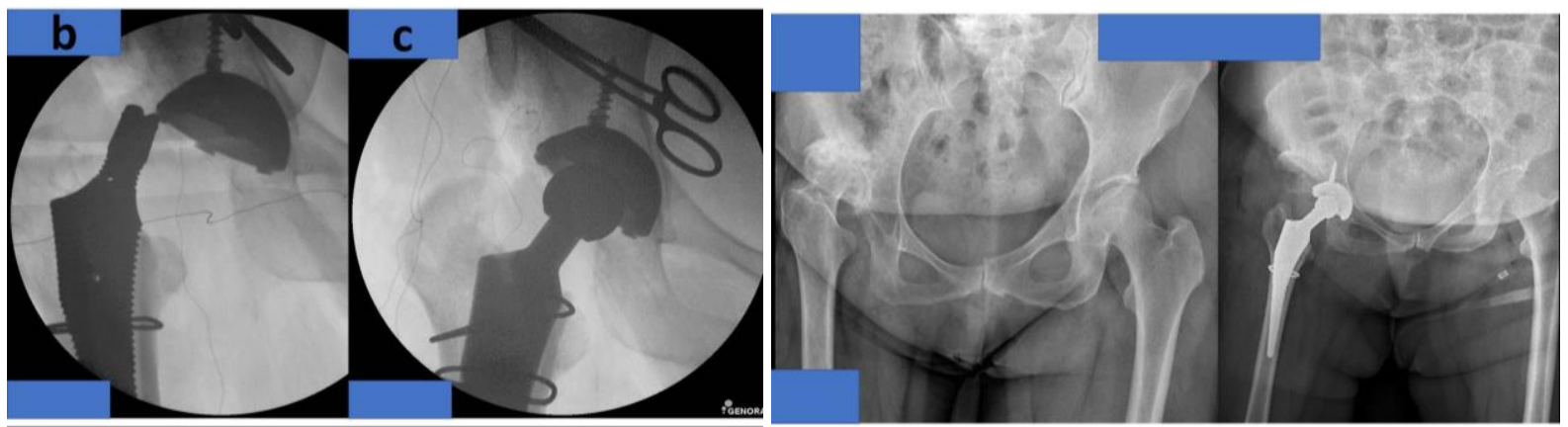

Fig 2: Case 1: Preoperative and postoperative radiographs 


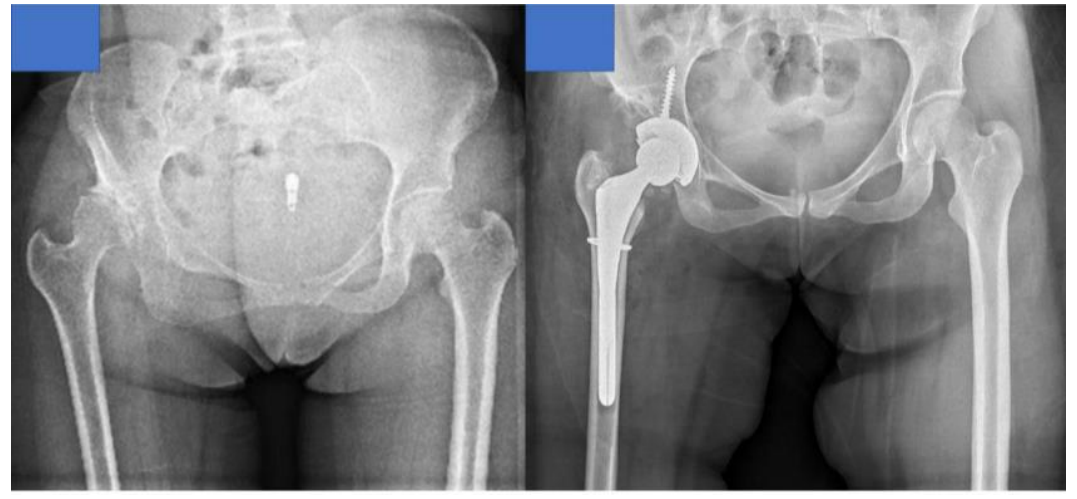

Fig 3: Case 2: Preoperative and postoperative radiographs

\section{References}

1. Crowe JF, Mani VJ, Ranawat CS. Total hip replacement in congenital dislocation and dysplasia of the hip. $\mathrm{J}$ Bone Joint Surg Am 1979;61(1):15.

2. Sarıkaya B, Ataoğlu B, Görmeli G, Öztürk BY, Turanlı $\mathrm{S}$. Eight-year follow-up of uncemented hydroxyapatite coated hip prosthesis for hip osteoarthritis secondary to developmental hip dysplasia. Eklem Hastalik Cerrahisi 2013;24(2):91-5.

3. Greber EM, Pelt CE, Gililland JM, Anderson MB, Erickson JA, Peters CL. Challenges in total hip arthroplasty in the setting of developmental dysplasia of the hip. J Arthroplasty 2017;32(9):38-44.

4. Hartofilakidis G, Stamos K, Karachalios T. Treatment of high dislocation of the hip in adults with total hip arthroplasty. J Bone Joint Surg Am 1998;80-A:510.

5. Krych AJ, Howard JL, Trousdale RT, Cabanela ME, Berry DJ. Total hip arthroplasty with shortening subtrochanteric osteotomy in Crowe type-IV developmental dysplasia. J Bone Joint Surg Am 2009;91A:2213.

6. Eskelinen A, Remes V, Ylinen P, Helenius I, Tallroth K, Paavilainen T. Cementless total hip arthroplasty in patients with severely dysplastic hips and a previous Schanz osteotomy of the femur. Acta Orthop 2009;80:263

7. Li H, Xu J, Qu X, Mao Y, Dai K, Zhu Z. Comparison of total hip arthroplasty with and without femoral shortening osteotomy for unilateral mild to moderate high hip dislocation. J Arthroplasty 2016;32(2):849-856.

8. Kawai T, Tanaka C, Ikenaga M, Kanoe H, Okudaira S. Total hip arthroplasty using Kerboull-type acetabular reinforcement device for rapidly destructive coxarthrosis. J Arthroplasty 2010;25:432-436.

9. Hartofilakidis G, Stamos K, Karachalios T. Treatment of high dislocation of the hip in adults with total hip arthroplasty. Operative technique and long-term clinical results. J Bone Joint Surg Am 1998;80(4):510-7.

10. Wu X, Li SH, Lou LM, Cai ZD. The techniques of soft tissue release and true socket reconstruction in total hip arthroplasty for patients with severe developmental dysplasia of the hip. Int Orthop 2012;36:1795-1801.

11. Lai KA, Shen WJ, Huang W, Chen MY. Cementless total hip arthroplasty and limb-length equalization in patients with unilateral Crowe type IV hip dislocation. J Bone Joint Surg Am 2005;87-A:339-345.

12. Yan F, Chen G, Yang L, He R, Gu L, Wang F. A reduction technique of arthroplasty without subtrochanteric femoral shortening osteotomy for the treatment of developmental high dislocation of hip: a case series of 28 hips. J Arthroplasty 2014;29(12):22892293.

13. Hartofilakidis G, Stamos K, Karachalios T. Treatment ofhigh dislocation of the hip in adults with total hip arthroplasty. J Bone Joint Surg Am 1998;80-A:510.

14. Dallari D, Pignatti G, Stagni CN, Giavaresi G, Del Piccolo N, Rani N. Total hip arthroplasty with shortening osteotomy in congenital major hip dislocation sequelae. Orthopedics 2011;34:e328-33.

15. Pagnano MW, Hanssen AD, Lewallen DG, Shaughnessy WJ. The effect of superior 327 placement of the acetabular component on the rate of loosening after total hip 328 arthroplasty. J Bone Joint Surg Am 1996;78A:1004-1014, 3292.

16. Zagra L, Bianchi L, Mondini A, Ceroni RG. Oblique femoral shortening osteotomy in 330 total hip arthroplasty for high dislocation in patients with hip dysplasia. Int Orthop 2015;39(9):1797-802.

17. Kayaalp ME, Can A, Erdogan F, Ozsahin MK, Aydingoz O, Kaynak G. Clinical and radiological results of Crowe type 3 or 4 dysplasia patients operated on with total hip arthroplasty using a cementless rectangular femoral component without fixating or grafting the transverse osteotomy site. J Arthroplasty 2020;35(9):2537-2542.

18. Tahta M, Isik C, Uluyardimci E, Cepni S, Oltulu I. Total hip arthroplasty without subtrochanteric femoral osteotomy is possible in patients with Crowe III/IV developmental dysplasia: total hip arthroplasty without femoral osteotomy. Arch Orthop Trauma Surg 2020;140(3):409-413.

19. Chen G, Nie Y, Xie J, Cao G, Huang Q, Pei F. Gait analysis of leg length discrepancy-differentiated hip replacement patients with developmental dysplasia: a midterm follow-up. J Arthroplasty 2018;33(5):14371441.

20. Benedetti MG, Catani F, Benedetti E, Berti L, Di Gioia A, Giannini S. To what extent does leg length discrepancy impair motor activity in patients after total hip arthroplasty? Int Orthop 2010;34(8):1115-21.

21. Eggli S, Hankelmayer S, Muller M. Nerve palsy after leg lengthening in total replacement arthroplasty for developmental dysplasia of the hip. J Bone Joint Surg Br 1998, 843.

22. Lai KA, Shen WJ, Huang LW, Chen MY. Cementless total hip arthroplasty and limb-length equalization in patients with unilateral Crowe type IV hip dislocation. J Bone Joint Surg Am 2005;87-A:339.

23. Kong X, Chai W, Chen J, Yan C, Shi L, Wang Y. Intraoperative monitoring of the femoral and sciatic nerves in total hip arthroplasty with high-riding 
developmental dysplasia. Bone Joint J 2019;101-B (11):1438-1446.

24. Li H, Yuan Y, Xu J, Chang Y, Dai K, Zhu Z. Direct leverage for reducing the femoral head in total hip arthroplasty without femoral shortening osteotomy for Crowe type 3 to 4 dysplasia of the hip. J Arthroplasty 2018;33(3):794-799. 